\title{
ON THE TORSION OF A CURVILINEARLY AEOLOTROPIC CYLINDER*
}

\author{
By R. K. BROWN AND E. E. JONES (University of Nottingham, England)
}

1. The general problem. It is well known that the problem of the torsion of a rectilinearly aeolotropic cylinder exhibiting elastic symmetry with respect to rightsections of the cylinder may be reduced to the Saint-Venant problem for the torsion of a certain isotropic cylinder [1, p. 193]. In this note it is shown that the torsion problem for a cylinder of general cross-section possessing curvilinear aeolotropy with respect to a triad of orthogonal curves at any point, two arbitrarily located parallel to the right sections and one parallel to the cylinder axis, may also be reduced to the SaintVenant torsion problem for materials which exhibit elastic symmetry of the types known as tetragonal, cubic, seven classes of the hexagonal system, and also for materials transversely isotropic with respect to lines parallel to the axis of the cylinder.

Rectangular Cartesian axes $x^{i}(i=1,2,3)$ are chosen with the $x^{3}$-axis along the axis of the cylinder, and the $x^{1}-x^{2}$ plane forming a right-section. The cylinder is formed from material which is elastically homogeneous and curvilinearly aeolotropic with respect to the orthogonal curves forming the axes of the curvilinear coordinate system defined by coordinates $\theta^{i}$, with corresponding metric tensor

$$
g_{i j}=x_{, i}^{\alpha} x_{, i}^{\alpha}
$$

where $x_{. i}^{\alpha}=\partial x^{\alpha} / \partial \theta^{i}$, and a repeated index implies summation over the values $1,2,3$ of that index. The strain energy function $W$ has the same form at all points when expressed in terms of the physical strain components $\gamma_{(i i)}$ of the infinitesimal strain tensor $\gamma_{i j}$ referred to the tangent lines to the $\theta^{i}$ coordinate curves [2]. In fact

$$
\gamma_{(i i)}=\gamma_{(i i)}=\gamma_{i j} /\left(g_{i i} g_{i j}\right)^{1 / 2} \quad \text { (no sum on } i, j \text { ) }
$$

and again if $e_{i i}$ are the components of the strain tensor referred to the $x^{i}$-axes, then

$$
\gamma_{i j}=x_{, i}^{\alpha} x_{, j}^{\beta} e_{\alpha \beta} .
$$

When the deformation is small in the classical sense, it may be assumed that at any point

$$
W=\frac{1}{2} E_{i j k l} \gamma_{(i i)} \gamma_{(k l)},
$$

where $E_{i j k l}$ are constants, exhibiting symmetry with respect to $i$ and $j, k$ and $l$, and the pairs $(i, j)$ and $(k, l)$. From (2), (3), (4) it follows that

$$
W=\frac{1}{2} C_{\alpha \beta \mu \nu} e_{\alpha \beta} e_{\mu \nu},
$$

where

$$
C_{\alpha \beta \mu \nu}=\sum_{i} \sum_{j} \sum_{k} \sum_{l} \frac{E_{i j k l} x_{. i}^{\alpha} x_{.}^{\beta} x_{k}^{\mu} x_{. l}^{\prime}}{\left(g_{i i} g_{i j} g_{k k} g_{l l}\right)^{1 / 2}}
$$

and $C_{\alpha \beta \mu \nu}$ has the same symmetry properties as $E_{i j k l}$.

*Received April 13, 1967. 
For subsequent convenience an alternative notation is introduced at this stage. The equations of the curves in the right-sections defining the aeolotropy are assumed to be parametrized, so that with $\theta^{1}=u, \theta^{2}=v$, we write

hence

$$
x^{1}=x=x(u, v), \quad x^{2}=y=y(u, v), \quad x^{3}=\theta^{3}=z,
$$

$$
\begin{array}{lll}
x_{, 1}^{1}=x_{u}, & x_{, 2}^{1}=x_{v}, & x_{, 3}^{1}=0, \\
x_{, 1}^{2}=y_{u}, & x_{, 2}^{2}=y_{0}, & x_{, 3}^{2}=0, \\
x_{, 1}^{3}=0, & x_{, 2}^{3}=0, & x_{, 3}^{3}=1,
\end{array}
$$

where $x_{u}=\partial x / \partial u$, etc., and again from (1)

$$
\begin{aligned}
& g_{11}=x_{u}^{2}+y_{u}^{2}, \quad g_{22}=x_{v}^{2}+y_{v}^{2}, \quad g_{33}=1, \\
& g_{13}=g_{31}=g_{32}=g_{23}=0, \quad g_{12}=g_{21}=x_{u} x_{v}+y_{u} y_{0} .
\end{aligned}
$$

The strain tensor $e_{i j}$ is defined in terms of the displacement gradients $\partial u_{i} / \partial x^{i}$ by

$$
e_{i j}=\frac{1}{2}\left(\partial u_{i} / \partial x^{j}+\partial u_{i} / \partial x^{i}\right),
$$

where for the torsion problem with small twist $\theta$ per unit length of the cylinder

$$
u_{1}=-\theta x^{2} x^{3}, \quad u_{2}=\theta x^{1} x^{3}, \quad u_{3}=\theta \phi\left(x^{1}, x^{2}\right),
$$

and $\phi$ is the warping function. In the notation of (7) the only non-zero components of $e_{i j}$ are

$$
e_{13}=\frac{1}{2} \theta(\partial \phi / \partial x-y), \quad e_{23}=\frac{1}{2} \theta(\partial \phi / \partial y+x) .
$$

The stress-strain relations derived from (5) are given by

so that from (10) we have

$$
\sigma_{i j}=\frac{1}{2}\left(\partial W / \partial e_{i i}+\partial W / \partial e_{i i}\right)=C_{\alpha \beta i j} e_{\alpha \beta},
$$

$$
\sigma_{i i}=C_{13 i i} e_{13}+C_{23 i} e_{23} .
$$

2. Materials with elastic symmetry. The cylinder is assumed to be made of material exhibiting elastic symmetry properties which include as special cases the tetragonal system, the cubic system, seven classes of the hexagonal system, and the system transversely isotropic with respect to lines parallel to the cylinder axis, $[3, \mathrm{p}$. 15]. These systems are characterized by the fact that the only nonzero components of $E_{i j k l}$ in (4) are subsets of the set $E_{1111}, E_{1122}, E_{2222}, E_{3333}, E_{1133}, E_{2233}, E_{1212}, E_{1211}, E_{1222}$, together with $E_{1313}=E_{2323}$ in each system. For such materials, by use of (6) and (8), we find that the only nonzero components of $\sigma_{i}$ in (11) are

$$
\begin{aligned}
\sigma_{13} & =C_{1313} e_{13}+C_{2313} e_{23}, \\
\sigma_{23} & =C_{1323} e_{13}+C_{2323} e_{23} .
\end{aligned}
$$

On substitution from (7)-(9) into (6), and considering the above nonzero components of $E_{i i k l}$, we have

$$
\begin{aligned}
& C_{1313}=2 A\left\{\frac{x_{u}^{2}}{x_{u}^{2}+y_{u}^{2}}+\frac{x_{v}^{2}}{x_{v}^{2}+y_{v}^{2}}\right\}, \quad C_{2313}=2 A\left\{\frac{x_{u} y_{u}}{x_{u}^{2}+y_{u}^{2}}+\frac{x_{v} y_{v}}{x_{v}^{2}+y_{v}^{2}}\right\}, \\
& C_{2323}=2 A\left\{\frac{y_{u}^{2}}{x_{u}^{2}+y_{u}^{2}}+\frac{y_{v}^{2}}{x_{v}^{2}+y_{v}^{2}}\right\},
\end{aligned}
$$


where $E_{1313}=E_{2323}=2 A$, a constant. But the curves defining the aeolotropy are orthogonal, so that $g_{i i}=0,(i \neq j)$, hence from (9) we have

$$
x_{u} x_{v}+y_{u} y_{v}=0,
$$

and in this case (13) reduce to

$$
C_{1313}=C_{2323}=2 A, \quad C_{2313}=0 .
$$

Immediately from (10) and (12) it follows that

$$
\sigma_{13}=A \theta(\partial \phi / \partial x-y), \quad \sigma_{23}=A \theta(\partial \phi / \partial y+x),
$$

as in the Saint-Venant problem, the elastic constant $A$ taking the place of the shear modulus of the corresponding isotropic cylinder. As usual these stress components satisfy the equilibrium equations with zero body force if $\nabla^{2} \phi=0$ in the right-section of the cylinder, and moreover lead to the usual boundary condition $\partial \phi / \partial n=n_{1} y-n_{2} x$ on the boundary of the section for zero force on the lateral surface of the cylinder, where $\left(n_{1}, n_{2}, 0\right)$ are the components of the unit outward normal to the boundary relative to the Cartesian coordinate axes. This is the Saint-Venant problem in the form of a Neumann boundary-value problem, solutions for which are known from many types of cylinder sections [1, Chapter 4].

It is interesting to note that the reduction to the Saint-Venant problem is possible independently of the geometry of the curves in the right section of the cylinder defining the aeolotropy, provided that these are orthogonal. The special case of cylindrical aeolotropy, when these curves are along the axial curves of a cylindrical polar coordinate system, has been referred to by Lekhnitskii [4, Chapter 4].

\section{REFERENCES}

1. I. S. Sokolnikoff, Mathematical theory of elasticity, 2nd ed., McGraw-Hill, New York, 1956

2. J. E. Adkins, Finite deformation of materials exhibiting curvilinear aeolotropy, Proc. Roy. Soc. A229 (1955), 119-134

3. A. E. Green and J. E. Adkins, Large elastic deformations, Oxford Univ. Press, 1960

4. S. G. Lekhnitskii, Theory of elasticity of an anisotropic elastic body, Holden-Day, San Francisco, 1963; translated by P. Fern 